\title{
Fundamento y aproximación Metodológica del Estudio de peces del Río San Pedro
}

\section{Basis and Methodological Approach for the Study of the San Pedro River Fish Fauna}

\author{
Evelyn Habit ${ }^{1}$ \& Oscar ParRa ${ }^{1}$ \\ 1 Unidad de Sistemas Acuáticos, Centro de Ciencias Ambientales EULA-Chile, Universidad de Concepción. Casilla \\ 160-C, Concepción, Chile. \\ E-mail: ehabit@udec.cl
}

\begin{abstract}
RESUMEN
El río San Pedro corresponde al sistema fluvial que desagua la cadena de ocho lagos andinos de la cuenca del río Valdivia (Región de los Ríos). Este sistema recorre $40 \mathrm{~km}$ entre el desagüe del lago Riñihue y su confluencia con el río Collileufu, donde cambia de nombre a río Calle Calle. En él se proyectó construir la "Central Hidroeléctrica San Pedro", cuyo muro se ubica $14 \mathrm{~km}$ aguas abajo del desagüe del lago Riñihue y la cual transforma 12,5 km de río en embalse. Ello generó la necesidad de elaborar un Estudio de Impacto Ambiental, el cual fue desarrollado por el Centro de Ciencias Ambientales Eula Chile (Universidad de Concepción). En relación a la ecología del río, dicho estudio puso especial énfasis en generar el conocimiento de detalle sobre su ictiofauna, con el fin de efectuar una evaluación de impactos especie específica. En este artículo presentamos los fundamentos y aproximaciones metodológicas para llevar a cabo el estudio intensivo, cuyos resultados dan origen a esta publicación monográfica y a la descripción de la distribución, abundancia, uso de hábitat, patrones de desplazamiento, relaciones de longitud-peso, primeros estadíos de vida y estructura genética de la ictiofauna del río San Pedro.
\end{abstract}

Palabras Clave: Río San Pedro, Central Hidroeléctrica, Evaluación de Impacto.

\begin{abstract}
The San Pedro River is the fluvial system being drained by the chain of eight Andean lakes in the Valdivia River Basin (de los Ríos Region). This system covers $40 \mathrm{~km}$ from the outlet of Riñihue Lake to its confluence with the Collileufu River, where it is renamed as Calle Calle River. In the San Pedro River, the "Central Hidroeléctrica San Pedro" (San Pedro Hydroelectric Plant) is planned, whose dam is located $14 \mathrm{~km}$ downstream from the outlet of the Riñihue Lake, which will transform $12.5 \mathrm{~km}$ of river in a reservoir. This generated the need for an Environmental Impact Assessment, which was developed by the Center for Environmental Sciences EULA-Chile (Universidad de Concepción). In relation to the river ecology, the study placed special emphasis on generating detailed knowledge about their ichthyofauna, in order to make a species-specific impact assessment. In this issue, we present the foundations and methodological approaches to undertake the intensive study, whose results give rise to this monograph and to the description of the distribution, abundance, habitat use, movement patterns, length-weight relationships, early life stages and genetic structure of the fish fauna of San Pedro River.
\end{abstract}

Keywords: San Pedro River, Hydroelectric plant, Environmental impact assessment.

\section{EL RÍO SAN PEDRO: SISTEMA FLUVIAL DE ALTA SINGULARIDAD Y RELEVANCIA AMBIENTAL}

Entre otros rasgos, Chile se caracteriza a lo largo de su territorio por la gran cantidad de ríos que lo atraviesan transversalmente desde la cordillera de los Andes hasta el litoral. Entre los ríos más importantes por el tamaño de sus cuencas, la longitud de sus cauces y el caudal de agua y sedimento transportado, están los ríos Loa, Aconcagua, Maipo, Maule, Biobío, Valdivia y Baker. El río San Pedro corresponde a uno de los tributarios del río Valdivia (Fig. 1). Existen variadas razones para considerar a este río como un sistema fluvial de alta singularidad. Este río corresponde al desagüe del Lago Riñihue, el cual a su vez es el último de una cadena de ocho lagos ubicados aguas arriba. Por tanto, corresponde a la descarga fluvial de uno de los sistemas 
de lagos norpatagónicos (también llamados Araucanos sensu Thomasson 1963), de mayor importancia ecológica y turística del país. Este sistema comprende los lagos Lácar (60 km², $630 \mathrm{msnm}$, ubicado en Argentina), Pirehueico (40 $\left.\mathrm{km}^{2}, 600 \mathrm{msnm}\right)$, Pellaifa $\left(7,2 \mathrm{~km}^{2}, 209 \mathrm{msnm}\right)$, Calafquén (120 km², $203 \mathrm{msnm})$, Pullinque $\left(5,8 \mathrm{~km}^{2}, 200 \mathrm{msnm}\right)$, Neltume (12 km², $186 \mathrm{msnm})$, Panguipulli $\left(117 \mathrm{~km}^{2}, 140\right.$ msnm), y Riñihue $\left(82,5 \mathrm{~km}^{2}, 117 \mathrm{msnm}\right)$. Este conjunto de sistemas lacustres se caracteriza por presentar una baja intervención humana, poseer condiciones oligotróficas a ultraoligotróficas, grandes profundidades y ser de origen glacial (Loeffler 1954; Thomasson 1963). Sus características limnológicas fueron estudiadas detalladamente por el grupo de trabajo del Dr. Hugo Campos (q.e.p.d) de la Universidad Austral de Chile, por lo que sus condiciones morfológicas, físicas, químicas y biológicas han sido descritas en diversos trabajos (e.g. Campos et al. 1987; Woelfl 1995; Campos et al. 2001; Woelfl et al. 2003).

Dado que este sistema interconectado de lagos, de régimen pluvio-nival, evacúa por un único sistema fluvial, el río San Pedro, esta regulado naturalmente por los lagos en su cuenca alta. Por lo mismo, es un río de aguas muy transparentes, ya que la mayor parte de la carga de sedimentos transportada desde la cuenca alta queda depositada en el fondo de los lagos. Aguas abajo, el río San Pedro da origen al río CalleCalle (luego de su confluencia con el río Collileufu), río que al unirse con el Cruces forma el Valdivia, en la ciudad del mismo nombre. Es decir, el río San Pedro es parte de una cuenca hidrográfica mayor, la del río Valdivia, de gran significado ambiental en Chile. El río San Pedro representa el 45\% del área de la cuenca del Valdivia. Sin embargo, contribuye con una proporción mucho mayor de sus caudales, ya que drena la parte más alta de la cuenca, con mayor pluviosidad. Por lo anterior, tiene gran relevancia hidrológica y ecológica en el comportamiento ambiental de la parte baja del sistema del río Valdivia, incluyendo su estuario. En términos biológicos, la presencia de conexiones a múltiples lagos aguas arriba, implica para el río San Pedro la posibilidad de albergar una mayor variedad de especies, ya que evacúa una cuenca con alta heterogeneidad de hábitats. Por último, se considera al río San Pedro de alta singularidad y relevancia ambiental, ya sea por sus características ecológicas y la biota que alberga y porque actualmente son muy pocos los sistemas fluviales que no presentan intervenciones antrópicas a lo largo del territorio chileno continental.

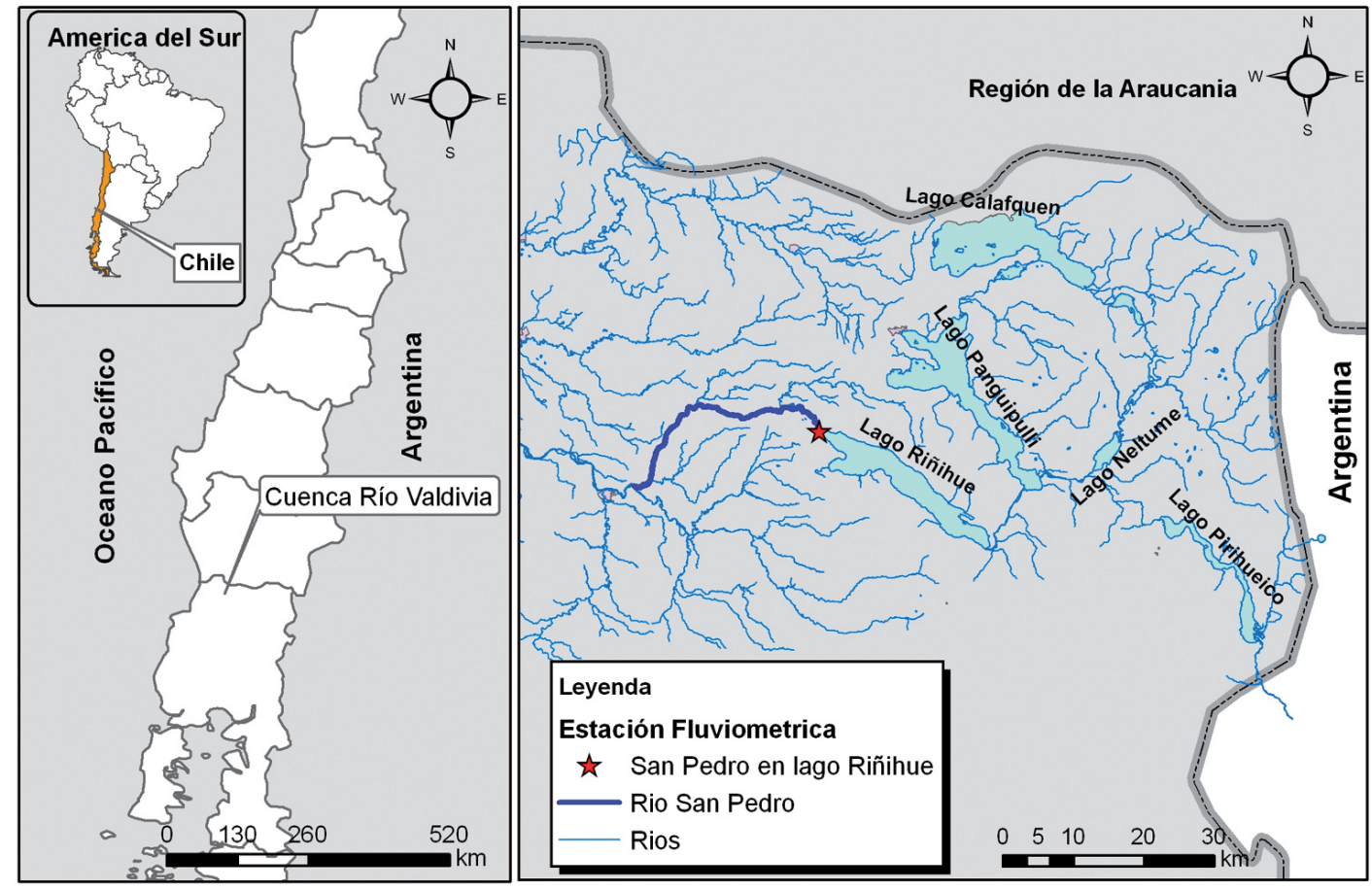

Figura 1. Ubicación de la cuenca del Río San Pedro.

Figure 1. San Pedro River localization. 


\section{IMPLICANCIAS ECOLOGICAS DE CENTRALES HIDROELECTRICAS}

Aún cuando los lagos norpatagónicos cuentan con información limnológica de base, el sistema fluvial del río San Pedro no tenía información de detalle. Por ello, cuando el Centro Eula fue solicitado para efectuar el Estudio de Impacto Ambiental de la Central Hidroeléctrica San Pedro (CHSP), proyectada en la parte alta del río San Pedro, se hizo ver al titular del proyecto (COLBUN S.A.) que por el valor de conservación de ese sistema fluvial, se debía efectuar un estudio de mucho detalle que permitiera profundizar y conocer aspectos de su estructura y dinámica hasta ahora desconocidos. En particular, resultaba relevante conocer aspectos específicos de la fauna íctica del río, la cual no sólo podría resultar impactada, sino que serviría como indicador del estado y valor ecosistémico de río (Cushman, 1985; Goodwin et al 2006; García et al 2011). Al respecto, la literatura científica reconoce múltiples impactos de centrales hidroeléctricas sobre la fauna de peces de los ríos. Uno de los principales es la interrupción del libre desplazamiento de organismos. La fragmentación impuesta por represas elimina la conectividad entre hábitats, generando un desequilibrio en la dinámica de metapoblaciones o poblaciones locales. La mayor parte de las especies de peces que habitan en sistemas fluviales complejos como la cuenca del Valdivia, establecen una dinámica metapoblacional, es decir funcionan como grupos de poblaciones locales que se conectan entre sí temporalmente por procesos de emigración e inmigración (Rieman \& Dunham 2000). Esto es, un sistema complejo de flujos de individuos entre sistemas lacustres y fluviales (y dentro de ellos) que pueden ser uni o bidireccionales, dependiendo de la capacidad de nado de cada especie y de las características propias del sistema. En otros términos, la dinámica espacial y temporal de las comunidades de peces en sistemas con redes hidrográficas complejas es también altamente compleja y variable en el tiempo. Los flujos migratorios, o desplazamientos locales activos (contra la corriente) o pasivos (a favor de la corriente), implican la mantención de un sistema en que cada población local puede servir de fuente o receptor de nuevos individuos, aportando a la mantención de la variabilidad genética total de la especie en una cuenca hidrográfica. Por ende, tal dinámica permite la persistencia de poblaciones que, aun cuando actúen temporalmente como locales (desconectadas entre lagos o entre un lago y el río), requieren de la mantención de tales flujos naturales de individuos a lo largo del tiempo.

Dependiendo de la historia de vida de los peces presentes en el área, la reducción de la conectividad puede resultar en efectos de distinto origen y magnitud. Para aquellas especies de hábitos migratorias (diádromas o que realizan largos viajes entre las aguas dulces y marinas para completar su ciclo de vida), la presencia de una barrera en su ruta migratoria representa un impacto relevante, pudiendo llevar al descenso en el número de individuos de la población y hasta su eventual extinción local. Por su parte, especies residentes en aguas continentales (lóticas o lénticas) que incluyen desplazamientos transversales o longitudinales necesarios para completar su ciclo de vida, pueden resultar afectadas localmente. Un ejemplo de ello son especies con adultos que habitan en la columna de agua en lagos o pozones de ríos, cuyos juveniles requieren de hábitats fluviales ribereños para desarrollarse. Por último, aún especies que no requieran desplazamientos obligatorios en su ciclo de vida, pueden resultar afectadas por la presencia de una barrera, la cual genera la interrupción del flujo génico entre poblaciones dentro de un mismo sistema. El flujo génico es un componente fundamental de la estructura poblacional de las especies porque determina hasta qué punto cada población local de una especie es una unidad evolutiva independiente. Si existe un importante flujo génico entre poblaciones locales, entonces todas las poblaciones están reproductivamente conectadas y evolucionan juntas. Sin embargo, si hay poco flujo génico cada población evoluciona en forma casi independiente (Slatkin 1994), y en tal caso la interrupción de la conectividad no constituye un problema para ellas. La fragmentación de poblaciones e interrupción del flujo génico puede entonces generar unidades sometidas a diferentes presiones selectivas $y$ acumulación de mutaciones o deriva génica, con la consecuente disminución de la variabilidad genética y aumento de la probabilidad de extinción local.

Tales impactos han sido reconocidos a nivel legal, y según lo indica la Ley No 18.892 de 1989 y sus modificaciones o Ley General de Pesca y Acuicultura en su Articulo No 168 "cuando se construyan represas en curso de agua fluviales que impidan la migración natural de los peces que en dichos cursos habitan con anterioridad a su construcción, será obligación de los propietarios de dichas obras civiles el efectuar un programa de siembra de dichas especies a objeto de mantener el nivel original de sus poblaciones, en ambos lados de la represa, o alternativamente construir las obras civiles que permitan dichas migraciones".

Otro impacto potencial significativo sobre la fauna de peces se debe a la fluctuación brusca de niveles del agua en zonas ribereñas, lo cual puede ocurrir según el tipo de operación de una central hidroeléctrica. Los hábitats ribereños someros son aquellos que contienen la mayor diversidad y abundancia de biota en sistemas fluviales de gran envergadura, como el río San Pedro. Por el contrario, el cauce principal, generalmente posee mayor velocidad de escurrimiento y profundidad, constituyendo un ambiente "pobre", ya que la mayoría de las especies no toleran tales condiciones por largos periodos. De esta forma, los ambientes someros de ribera concentran una alta productividad y áreas de 
refugio para los estadios de vida más lábiles (o con mayor probabilidad de mortalidad por bajo nivel de tolerancia a situaciones de estrés), tales como huevos, larvas y juveniles de peces. A su vez, son estos mismos estadíos los que presentan la menor capacidad de desplazamiento, lo cual los hace altamente vulnerables a cambios bruscos en su hábitat. Lo anterior también es válido para peces nativos de hábitos bentónicos, los cuales buscan refugio en áreas intersticiales durante bajas bruscas de caudal. En este caso, si el nivel de agua no se recupera en un corto periodo, los peces mueren, evento que puede resultar masivo, tal como fue registrado en operaciones de apertura de canales de riego en la cuenca del Río Itata (Habit \& Parra 2001). Finalmente, la alteración de una o todas las poblaciones locales de peces de un área impactada por barreras, genera cambios a nivel de la comunidad (e.g., cambios en la proporción de especies o diversidad en el área), que pueden tener repercusiones en la estructura trófica y dinámica general del sistema acuático (García de Jalon et al. 1993).

En este sentido, la Ley de Bases del Medio Ambiente y su Reglamento, establecen la necesidad de efectuar estudios de Líneas de Base que permitan hacer una adecuada predicción de impactos, así como una efectiva propuesta de medidas de mitigación-compensación para proyectos hidroeléctricos. Sin embargo, conocer la estructura y dinámica de poblaciones y comunidades de peces de grandes ríos como el San Pedro, representaba un desafío mayor, ya que el sólo muestreo puntual y esporádico de esta fauna no sería representativo de los procesos que se requería conocer. En este sentido, se consideró que una evaluación genérica de impactos ambientales resultaría inadecuada, necesitando generar la información apropiada para responder de qué magnitud e importancia resultarían los impactos de la Central San Pedro sobre la comunidad de peces del mismo. Para ello, se plantearon cuatro interrogantes básicas: (a) ¿Cuáles son los principales desplazamientos longitudinales y horizontales que efectúan las especies nativas en el área?, (b) ¿cuál es la dinámica del uso de hábitats ribereños y qué significado ecológico particular tienen para las distintas especies?, (c) ¿existe flujo génico entre las poblaciones del Lago Riñihue, río San Pedro y aquellas ubicadas aguas abajo?, (d) ¿cuál es la abundancia relativa de la fauna nativa en relación a la introducida?

\section{CRITERIOS Y ACCIONES PARA ABORDAR EL ESTUDIO AMBIENTAL DE ESTE SISTEMA FLUVIAL COMPLEJO}

Responder las interrogantes planteadas implicaba un desafío no asumido hasta el momento por ningún otro proyecto hidroeléctrico, ya que abordar el estudio de sistemas complejos requiere de aproximaciones metodológicas innovadoras. Por ello, para llevar a cabo el estudio de la fauna de peces del sistema fluvial San Pedro se implementaron los siguientes criterios y acciones:

1) El estudio incluyó como especies objetivo primario a las especies nativas de agua dulce de Chile presentes en el sistema fluvial del río San Pedro. Ello quiere decir que las especies introducidas, tales como los salmonídeos (truchas), no se incluyeron en estudios específicos de migraciones o uso de hábitat. Las principales razones para seguir este criterio fueron: (a) Las especies nativas representan nuestra biodiversidad y como tal son los objetos de conservación primarios. (b) Las especies salmonídeas, dado que son introducidas, no tienen valor de conservación per se, sino que mas bien tienen un valor de uso o comercial, debido a que sustentan la actividad de pesca deportiva. De esta forma, el interés de mantener las poblaciones de salmonídeos en un área es más bien de carácter social. (c) Las especies de salmonídeos han sido definidas por IUCN (www.iucn.org) como las especies invasoras de agua dulce más ampliamente distribuidas en el planeta, estando clasificadas las dos especies presentes en el río San Pedro (Oncorhynchus mykiss o trucha arcoiris y Salmo trutta o trucha café) entre las 100 "especies invasivas más dañinas del planeta" ("World's Worst Invasive Alien Species" sensu IUCN). Por ello, en un contexto de conservación, resulta más apropiado evitar su proliferación en un área donde son poco abundantes.

2) Se definieron áreas de estudio de distinto nivel de detalle según las preguntas a contestar. Cada pregunta se abordó de acuerdo a la especificación requerida para obtener una respuesta adecuada. Es así como algunos análisis, entre ellos los genéticos requirieron de muestreos no sólo en la cuenca del río Valdivia, sino que también en cuencas vecinas. De igual forma, para comprender el valor y singularidad de la fauna de peces del río San Pedro se realizaron estudios que abarcaron la cuenca completa del río Valdivia. Por último, para responder preguntas más acotadas a los usos de hábitat, desplazamientos entre ellos y entre zonas del río San Pedro, se utilizó como área de estudio el tramo comprendido entre el desagüe del Lago Riñihue y su confluencia con el río Quinchilca, es decir aproximadamente $45 \mathrm{Km}$ de longitud.

Las repetidas observaciones en terreno, asociadas al muestreo de peces, permitieron determinar la ocurrencia de tres tipos de macrohábitat para peces, o macrozonas, las cuales se definieron en función de sus características hidrogeomorfológicas. Esto es a la escala de tramos, es decir, aquella que determina a grandes rasgos las características del macrohábitat disponible para la flora y fauna acuática y ribereña (Fig. 2):

Macrozona 1: Constituida por la parte terminal y el desagüe del lago Riñihue, hasta el sector de Piedra Blanca (Piedra 
Comedor). Este tramo consiste principalmente de sectores lacustres de menor profundidad, incorporando también 1,5 $\mathrm{km}$ de río, que corresponden a los inicios del San Pedro. Se caracteriza por ser un tramo de pendiente contraria, con un escurrimiento profundo, sin rápidos. En esta zona se establecieron 9 sitios de estudio (Fig. 2; Tabla 1), en los cuales se muestreó preferentemente sitios en la orilla del lago e inicio del río, correspondientes a ambientes someros con poca velocidad de corriente y con vegetación acuática enraizada.

Macrozona 2: Este tramo es el de mayor extensión; con una longitud aproximada de $30 \mathrm{~km}$, contempla aproximadamente el $75 \%$ del desarrollo longitudinal del río San Pedro. Se caracteriza por ser un tramo de río no-aluvial, encajonado, y con una pendiente de lecho mayor que en la macrozona 1 . La pendiente resulta en mayores velocidades de escurrimiento, observándose secuencias de rápidos profundos y pozas en los cuales prevalecen los sustratos de bolones, con bloques de grandes dimensiones. En algunos tramos, hay una planicie de inundación angosta, poco desarrollada. Este tramo se localiza entre el sector de Los Bajos hasta Chacaipulli (Fig. 2, Tabla 1) sumando un total de 17 sitios de muestreo.

Macrozona 3: Corresponde a un tramo de río aluvial, con secuencias de pozones y rápidos. El río no escurre encajonado, sino que muestra una amplia planicie de inundación, de hasta $600 \mathrm{~m}$ de ancho, en su ingreso al Valle Central existiendo una mayor longitud entre las dos riberas Norte-Sur por lo tanto los tramos de rápidos fuertes ya disminuyen. Este tramo de $8,5 \mathrm{~km}$ de longitud se ubica desde el sector del Balsadero hasta aproximadamente $200 \mathrm{~m}$ aguas abajo de la Confluencia con el río Quinchilca. (Fig. 2,
Tabla 1). En él fue posible establecer 9 sitios de muestreo, dentro de los cuales hubo sitios con hábitats de bolones, arenosos, y también con vegetación.

3) Se diseñó una estrategia de muestreo intensiva en el tiempo y con una alta densidad de estaciones de muestreo. En este estudio, se llevaron a cabo muestreos en las cuencas de los ríos Toltén (6 sitios), Valdivia (87 sitios) y Bueno (2 sitios), en un total de 416 días efectivos de muestreo. Sólo en la cuenca del río San Pedro se establecieron 35 sitios de muestreo, los cuales fueron muestreados en promedio 2 a 5 veces al mes. El estudio de campo se extendió entre los meses de octubre de 2005 a febrero de 2007 y junio de 2007 a febrero de 2008. Es decir, un total de 26 meses efectivos en el campo, de los cuales, el $73 \%$ del tiempo se estuvo muestreando en terreno.

4) Se implementaron todas las técnicas de muestreo apropiadas para registrar la diversidad del sistema en su totalidad. Dos premisas básicas se siguieron en este estudio. En primer lugar, realizar muestreos con devolución, es decir, evitar al máximo la mortalidad debida a muestreo. Para ello, todos los artes de pesca se utilizaron de tal forma de reducir la mortalidad, a su vez que, todos los peces capturados fueron mantenidos en buenas condiciones fuera de su hábitat (recipientes aireados y a baja temperatura) y anestesiados para realizar procedimientos de mediciones y marcaje. En segundo lugar, se tuvo como premisa abarcar todos los tipos de hábitats (a escala de micro y macrohábitats) de tal forma de representar adecuadamente el uso espacial y temporal de todos los estadíos de vida (huevos, larvas, reclutas, juveniles y adultos).

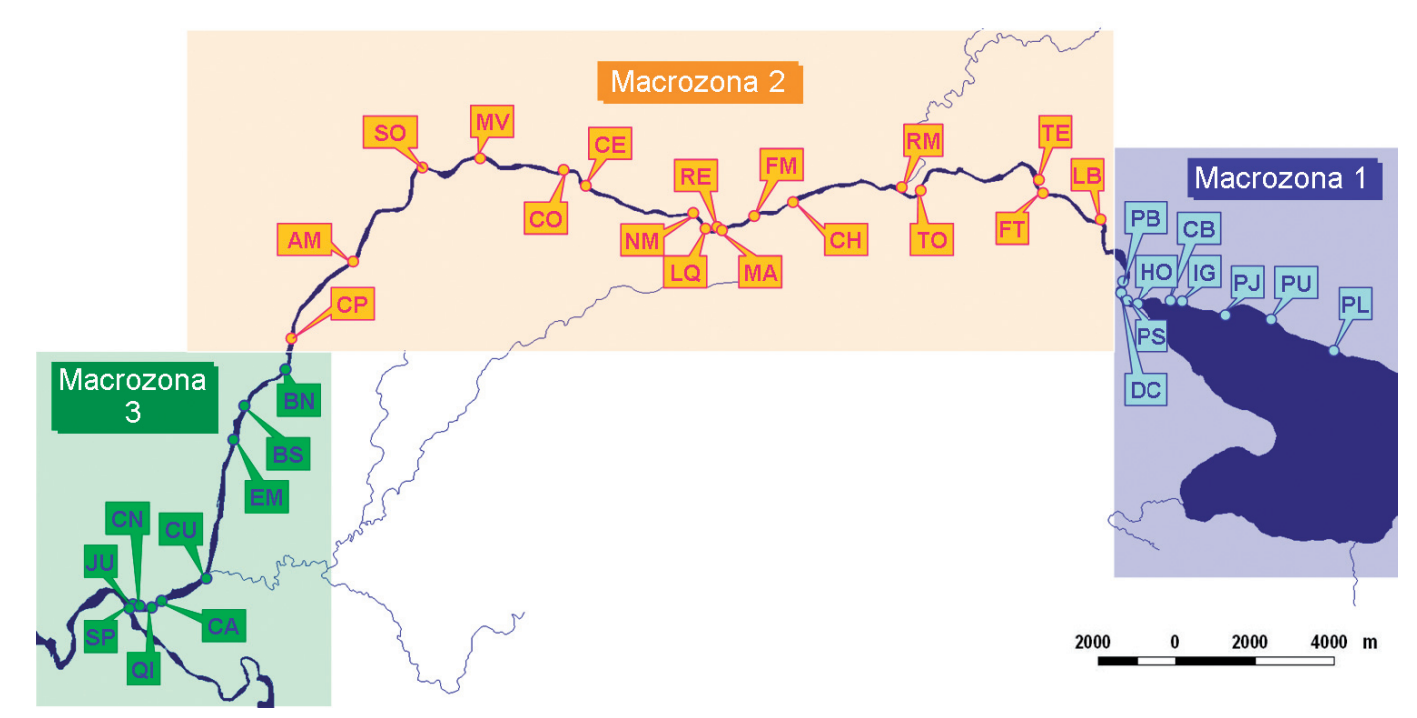

Figura 2. Mapa del Río San Pedro indicando las tres macrozonas hidrogeomorfológicas, con sus correspondientes sitios de muestreo.

FIGURE 2. San Pedro River map showing the three hydrogeomorphological macrozones, and sampling sites. 
Tabla 1. Ubicación geográfica de los sitios de muestreo en cada macrozona, ordenados desde el Lago Riñihue hacia aguas abajo, y expresados en coordenadas UTM. Datum WGS84, Huso 19.

Table 1. Geographic localization of each sampling site from the Riñihue Lake downstream. Coordinates in UTM. Datum WGS84, Huso 19.

\begin{tabular}{|c|c|c|c|c|c|}
\hline $\begin{array}{c}\text { Macrozona } \\
\text { hábitat }\end{array}$ & Sitio & $\mathbf{N}^{0}$ Correlativo & Código & $\mathbf{X}$ & $\mathbf{Y}$ \\
\hline \multirow[t]{9}{*}{1} & Playa & 1 & PL & 722328 & 5593120 \\
\hline & Puntilla & 2 & PU & 721189 & 5593820 \\
\hline & Picá de Javier & 3 & PJ & 719743 & 5593884 \\
\hline & Isla Grande & 4 & IG & 718328 & 5594046 \\
\hline & Playa Sur & 5 & PS & 717940 & 5593975 \\
\hline & Cabañas & 6 & $\mathrm{CB}$ & 718198 & 5594178 \\
\hline & Hotel & 7 & $\mathrm{HO}$ & 717859 & 5594106 \\
\hline & Desembocadura Catalina & 8 & $\mathrm{DC}$ & 717642 & 5594034 \\
\hline & Piedra Blanca & 9 & PB & 717526 & 5594451 \\
\hline \multirow[t]{17}{*}{2} & Los Bajos & 10 & LB & 716917 & 5595892 \\
\hline & Carmen del Trafún Este & 11 & $\mathrm{TE}$ & 715426 & 5596941 \\
\hline & Fundo Tornagaleones & 12 & FT & 715400 & 5596832 \\
\hline & Carmen del Trafún Oeste & 13 & TO & 712171 & 5597029 \\
\hline & Río Mañío & 14 & $\mathrm{RM}$ & 711860 & 5597161 \\
\hline & Champulli & 15 & $\mathrm{CH}$ & 708350 & 5596457 \\
\hline & Forestal Maitén & 16 & FM & 707795 & 5596191 \\
\hline & El Maitén & 17 & MA & 707240 & 5596014 \\
\hline & Represa & 18 & $\mathrm{RE}$ & 707146 & 5596057 \\
\hline & La Quinta & 19 & LQ & 706945 & 559575 \\
\hline & Nuevo Maitén & 20 & $\mathrm{NM}$ & 706548 & 5596095 \\
\hline & Cun-Cun Este & 21 & $\mathrm{CE}$ & 703554 & 5597017 \\
\hline & Cun-Cun Oeste & 22 & $\mathrm{CO}$ & 703088 & 5597452 \\
\hline & Malihue Viejo & 23 & MV & 700691 & 5597921 \\
\hline & Santa Olga & 24 & SO & 699317 & 5597654 \\
\hline & Anita María & 25 & $\mathrm{AM}$ & 697222 & 5595025 \\
\hline & Chacaipulli & 26 & $\mathrm{CP}$ & 695988 & 5593261 \\
\hline \multirow[t]{9}{*}{3} & Balsa Norte & 27 & $\mathrm{BN}$ & 695694 & 5592383 \\
\hline & Balsa Sur & 28 & BS & 694813 & 5591543 \\
\hline & El Mosqueto & 29 & EM & 694546 & 5590769 \\
\hline & Cuyincahuin & 30 & $\mathrm{CU}$ & 693429 & 5586939 \\
\hline & Camping & 31 & $\mathrm{CA}$ & 692278 & 5586120 \\
\hline & San Pedro & 32 & SP & 691787 & 5586085 \\
\hline & Quinchilca & 33 & QI & 692029 & 5586006 \\
\hline & Junta & 34 & $\mathrm{JU}$ & 691621 & 5586199 \\
\hline & Cuyincahuin Nuevo & 35 & $\mathrm{CN}$ & 692002 & 5586058 \\
\hline
\end{tabular}


5) Se construyó un laboratorio específico, con la instrumentación y el personal necesario, en el área de estudio para desarrollar las actividades de campo.

\section{ESTACION ECOLOGIA SAN PEDRO}

Sobre la base de la relevancia de los aspectos ambientales de este sistema fluvial, la empresa COLBUN S.A. construyó una Estación Ecológica o Laboratorio ubicado en el Fundo Santa Olga, ribera norte del río, sector Malihue Viejo. Esta estación ecológica tiene por objetivos:

a) Disponer y albergar la infraestructura y equipamiento adecuados para efectuar los estudios de línea de base limnológicos.

b) Permitir el desarrollo de un estudio intensivo y de largo plazo sobre patrones de desplazamiento y uso de hábitat de peces nativos.

c) Permitir el desarrollo de los programas de seguimiento indicados en la Resolución de Calificación Ambiental del Proyecto.

d) Albergar al equipo de profesionales a cargo de las actividades de investigación y visitantes.

e) Servir como una instancia de extensión y para facilitar la relación con los habitantes de la zona y actores relevantes del área, tales como pescadores deportivos y representantes locales y visitantes.

La Estación Ecológica (Figs. 3 y 4) comenzó a funcionar el día 5 de enero del 2006 y cuenta con la siguiente infraestructura, equipamiento:

- Una casa habitación para seis personas residentes permanentes.

- Un Laboratorio húmedo equipado con estanques, acuarios, línea de agua filtrada y desinfectada, y línea de agua sin filtrar para la mantención de peces marcados o sometidos a cirugías de implante de transmisores.

- Estanques exteriores de 500 L para la recuperación de los peces.

- Laboratorio seco equipado con computadores, impresoras, microscopios, estereomicroscopios, balanzas digitales y material fungible, para análisis de detalle.

- Bodega de almacenamiento de equipos y materiales de terreno, tales como equipos de pescas eléctrica, bote con motor fuera de borda, redes monofilamento, redes de plancton, redes surber, balanzas digitales portátiles, radios portátiles e ictiómetros.

- Un hall de recepción de estudiantes o personas interesadas en conocer los estudios en desarrollo.



Figura 3. A) Casa habitación estación ecológica San Pedro. B) Vista externa laboratorio.

Figure 3. A) House of the San Pedro ecological station. B) Outside view of the lab. 



Figure 4. A) Bodega; B) Laboratorio seco, C-E) Laboratorio húmedo, F) Laboratorio con estanques 500L.

Figure 4. A) Stockroom; B) Dry lab; C-E) Wet lab; F) Lab with 500 L tanks.

Se conformó un grupo de trabajo especializado, constituido por profesionales que trabajaron y habitaron directamente en el área de estudio por un período de dos años y medio, más un grupo de trabajo en la Universidad de Concepción. Todo el equipo operó a cargo de un Jefe de Proyecto y Responsable Científico de la Estación, seis profesionales Biólogos, residentes permanentes de la estación y un investigador más dos técnicos encargados del área de estudios genéticos.

Se establecieron nexos con la comunidad local y actores relevantes para involucrarlos en el estudio y sus alcances. A su vez, la estación fue y seguirá siendo utilizada para la formación de recursos humanos, a través de cursos programados con dichos fines. Por último, esta Estación juega un rol importante en los aspectos de extensión del proyecto y de contacto con la comunidad local e interesada en la protección ambiental del sistema ecológico del río San Pedro y su entorno. Las proyecciones de este laboratorio incluyen su mantención para efectuar el Programa de Seguimiento Ambiental que incluye un programa de monitoreo permanente de las comunidades biológicas y calidad del agua del sistema fluvial San Pedro.

La estrategia planteada para desarrollar este estudio de peces permitió generar una gran cantidad de información inédita sobre los peces de agua dulce nativos, los cuales son entregados a la comunidad científica y en general al público interesado a través de esta publicación. 


\section{AGRADECIMIENTOS}

Agradecemos a COLBUN S.A. por el financiamiento de este estudio y al proyecto DIUC Semilla Patagonia 210.310.0571SP por el financiamiento de la publicación.

Agradecemos a Alejandra Stehr y Priscila Piedra por la elaboración de las figuras 1 y 2 , respetivamente.

\section{BIBLIOGRAFÍA}

Campos, H., W. Steffens, G. Agüero, O. Parra \& L. Zúñoga. 1987. Limnology of Lake Riñihue. Limnológica 18(2): 339-345.

Campos, H., Hamilton, D.P., Villalobos, L., Iberger, J. \& JAVAM, A. 2001. A modelling assessment of potential for eutrophication of Lake Riñihue, Chile. Archiv für Hydrobiologie 151: 101-125.

Cushman, R.M. 1985. Review of ecological effects of rapidely varying flows downstream from hydroelectric facilities. North American journal of fisheries Management 5:330339.

García, A., Jorde, K., Habit E., CaAmaño, D. \& Parra O. 2011. Downstream environmental effects of dam operations: changes in habitat quality for native fish species. River Research Applications 27: 312-327.

Goodwin, P., Jorge, K., Meier, C. \& Parra, O. 2006. Minimizing environmental impacts of hydropower development: transfering lessons from past projects to a proposed strategy for chile. Journal of hydroinformatics 8: 253-270.

García de Jalón, D., Mayo, M. Hervella, F., Barcelo, E. \& Fernández, T. 1993. Principios y técnicas de gestión de la pesca en aguas continentales. Mundi-Prensa. Madrid. 247 pp.

Habit, E. \& PARra, O. 2001. Impacto ambiental de los canales de riego sobre la fauna de peces fluviales. Ambiente y Desarrollo 7(3): 50 -56.

LÖFFLER, H., 1954. Arbeitsbericht der limnologischen Andenexpedition 1953/54. Mitteilungen Geographische Geselschaft 96: 329-331.

Rieman, B. \& Dunham, J. 2000. Metapopulations and salmonids: A synthesis of life history patterns and empirical observations. Ecology Freshwater Fish 9: 51-64.

Slatkin, M. 1994. Gene flow and population structure. Ecological Genetics. LA Real,. Princeton University Press. Vol I. 3$17 \mathrm{pp}$.

Thomasson, K. 1963. Araucanian Lakes. Plankton studies in North Patagonia with notes on terrestrial vegetation. Acta Phtytogeographica Suecica 47: 1- 141.

WoElfl, S. 1995. Untersuchugen zur Zooplanktonstruktur einschliesslich der mikrobiellen Gruppen unter besonderer Berücksichtigung der mixotrophen Ciliaten in zwei südchilenischen Andenfusseen. Ph.D. Thesis, Faculty for Biology, University of Constance, Germany, Hartung Gorre Verlag Konstanz, Germany. 242 pp.

Woelfl, S., Villalobos, L. \& Parra, O. 2003. Trophic parameters and method validation in Lake Riñihue (North Patagonia, Chile) from 1978-1997. Revista Chilena de Historia Natural 76(3): 459-474.

Recibido: 02.01.12

Aceptado: 27.02.12 